\title{
Publisher's Note: Observation of Optomechanical Strain in a Cold Atomic Cloud [Phys. Rev. Lett. 119, 163201 (2017)]
}

Noam Matzliah, Hagai Edri, Asif Sinay, Roee Ozeri, and Nir Davidson

(Received 23 October 2017; published 31 October 2017)

DOI: 10.1103/PhysRevLett.119.189902

This paper was published online on 19 October 2017 with a typographical error in Eq. (1). Equation (1) should read as

$$
\begin{aligned}
\vec{f}_{\mathrm{es}} & =\frac{\hbar \Gamma^{2}}{8 \Delta} \frac{I}{I_{\mathrm{s}}} \frac{\vec{\nabla}_{\perp} n}{n}=-\vec{\nabla}_{\perp} U_{\mathrm{es}} \\
U_{\mathrm{es}} & =-\frac{\hbar \Gamma^{2}}{8 \Delta} \frac{I}{I_{\mathrm{s}}} \ln \left(\frac{n}{n_{0}}\right)
\end{aligned}
$$

The equation has been corrected as of 23 October 2017. The equation is correct in the printed version of the journal. 